\title{
Isotropic magnetoresistance of icosahedral Al-Pd-Mn
}

\author{
M. Rodmar \\ Fasta Tillståndets Fysik, Kungliga Tekniska Högskolan, SE 10044 Stockholm, Sweden \\ B. Grushko, N. Tamura, * and K. Urban \\ Institut für Festkörperforschung, Forschungszentrum Jülich GmbH, D-52425 Jülich, Germany \\ Ö. Rapp \\ Fasta Tillståndets Fysik, Kungliga Tekniska Högskolan, SE 10044 Stockholm, Sweden
}

(Received 23 March 1999)

\begin{abstract}
The temperature dependence of the electrical resistivity $\rho(T)$ and the magnetoresistance $\Delta \rho(B, T) / \rho(0, T)$ have been studied along the twofold and fivefold symmetry directions of an icosahedral Al-Pd-Mn in a high quality monograin crystal. To control the influence of any magnetic contribution, samples from the transversal and axial extremes of the crystal were measured along both the twofold and fivefold symmetry directions. The temperature dependence of the resistivity and the magnetoresistance were found to be isotropic. $\rho(T)$ and $\Delta \rho(B, T) / \rho(0, T)$ varied in a random way in the transversal direction of the ingot, while systematic variations were observed along the growth direction. These observations could be qualitatively correlated to a gradient of the $\mathrm{Mn}$ concentration of below $0.2 \% / \mathrm{cm}$ in the growth direction of the crystal. [S0163-1829(99)10433-8]
\end{abstract}

\section{INTRODUCTION}

The focus of experimental investigations of quasicrystals has been on polygrained samples since the beginning in 1984. ${ }^{1}$ Consequently the question of the possibility of anisotropic properties of icosahedral quasicrystals has seldom been addressed. More recently however, large single grain samples of icosahedral ( $i$ )-Al-Pd-Mn have become available, which allow for transport measurements along different symmetry axes. A handful of reports have been published on the electronic transport properties of this material. ${ }^{2-6}$ However, these results show large differences between the observed magnitude of both the resistivity and the magnetoresistance. For instance, Yokoyama et al. ${ }^{2}$ reported that the resistivity at $4 \mathrm{~K}$ was about $70 \%$ larger along the twofold axis than along the fivefold one, and the magnetoresistance (MR) at $4 \mathrm{~K}$ and $8 \mathrm{~T}$ in $i-\mathrm{Al}_{70} \mathrm{Pd}_{21} \mathrm{Mn}_{9}$ was found to be $3.3 \%$ in Ref. 3 and about three times smaller in Ref. 4. Furthermore, in Ref. 5, the MR of $i-\mathrm{Al}_{70.5} \mathrm{Pd}_{21.1} \mathrm{Mn}_{8.4}$ at $4 \mathrm{~K}$ and $8 \mathrm{~T}$ was larger along the fivefold direction than along the twofold direction by about $10 \%$, while in Ref. 6 the MR along the fivefold axis was instead smaller by a factor of similar magnitude.

As noted already in these papers, ${ }^{2-6}$ the influence of the Mn concentration on the transport properties of $i$-Al-Pd-Mn may cause difficulties. Only a small fraction of Mn sites in the quasicrystal are magnetic with a magnetic moment which decreases with decreasing Mn concentration and appears to vanish below about 9 at. \% Mn. ${ }^{7}$ However, also below this limit the low-temperature paramagnetism is strongly dependent on Mn concentration. ${ }^{8}$ Furthermore, minute precipitates of Mn-rich phases, extrinsic to the icosahedral phase, could have a strong influence on the transport properties. Our previous investigations have been performed on samples at the present state of the art of single grain quasicrystal growing, and in particular, have been found to be homogenous with respect to manganese concentration to within $0.2 \mathrm{wt} \%$ in sample pieces of 1-cm length from different parts of the ingot. ${ }^{6}$ The problem is thus to determine if $\mathrm{Mn}$ concentration gradients below about $0.2 \% / \mathrm{cm}$ can influence the measured properties or if the magnetoresistance of $i-\mathrm{Al}-\mathrm{Pd}-\mathrm{Mn}$ is anisotropic.

Since the variation of manganese concentration of below $0.2 \%$ is below the detection limit our analyses methods, including ICP-OES, (inductively coupled plasma optical emission spectra), and EDX (energy dispersive X-ray analyses) in SEM (scanning electron microscope), it is clear that samples from different locations of the ingot must be examined. We have cut a series of samples along the twofold and fivefold symmetry axes from various parts of an ingot of single grain $i$-Al-Pd-Mn in such a way that transport properties along a particular symmetry direction can be followed through the ingot. The resistivity and magnetoresistivity were measured. We find systematic variations in both quantities as a function of position in the sample, up to a factor of 2 in the magnetoresistance at $1.5 \mathrm{~K}$ and $12 \mathrm{~T}$. It is concluded that the differences observed are mainly due to variation of Mn concentration in the sample at the level below about $0.2 \%$, and that the magnetoresistance of $i-\mathrm{Al}-\mathrm{Pd}-\mathrm{Mn}$ is apparently isotropic.

\section{EXPERIMENTAL DETAILS}

The sample material was taken from the central part of an icosahedral crystal grown by the Czochralski technique from the alloy of $\mathrm{Al}_{72.7} \mathrm{Pd}_{20.2} \mathrm{Mn}_{7.1}$. In this technique the crystal grows from the melt by solidification of the surface of a crystalline seed, which is dipped into the melt and subsequently slowly pulled away from it. ${ }^{9}$ In this case the composition of the icosahedral crystal is different from that of the initial alloy due to the peritectic character of $i$-Al-Pd-Mn formation. ${ }^{10}$ Coarsely speaking, for a successful growth the alloy composition should contain about two atomic percent 


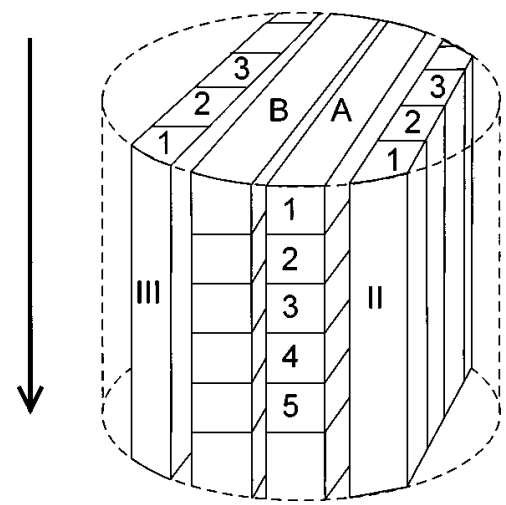

FIG. 1. The original crystal and the positions of the samples. The crystal is about $10-\mathrm{mm}$ long and $10 \mathrm{~mm}$ in diameter. The growth direction of the crystal is along the arrow. Notations for samples studied are $n \mathrm{II}$, and $n \mathrm{III}, n=1,2,3$ for samples along the twofold direction, and $n \mathrm{~A}, n=1-5$ for the fivefold axis. In addition, several measurements were made along the length of individual samples as described in the text. The stacks, III, B, A, and II are parallel. Samples $n$ III are perpendicular to samples $n$ A.

more Al than the resulting icosahedral crystal. Due to the limited volume of the molten alloy, its composition and the composition of the grown crystal change during the processing. As mentioned, the compositional variations of a single grain $i$-Al-Pd-Mn are quite small under the usual growth conditions of this phase. However, systematic investigations of a series of crystals grown by this technique have shown that they are possible and that $\mathrm{Pd}$ concentration varies less than that of $\mathrm{Al}$ and $\mathrm{Mn}$. As an example, a crystal grown from $\mathrm{Al}_{72.4} \mathrm{Pd}_{20.6} \mathrm{Mn}_{7.1}$ gave $\mathrm{Al}_{70.1} \mathrm{Pd}_{21.5} \mathrm{Mn}_{8.4}$ and from $\mathrm{Al}_{73.3} \mathrm{Pd}_{20.1} \mathrm{Mn}_{6.6}$ gave $\mathrm{Al}_{71.9} \mathrm{Pd}_{21.0} \mathrm{Mn}_{7.1}$ in ICP-OES measurements. ${ }^{11}$ Since the crystal thus contains less $\mathrm{Al}$ and more $\mathrm{Mn}$ than the initial alloy, the Al concentration should increase and that of $\mathrm{Mn}$ should decrease during the crystal growth. In the direction from top to bottom of the crystal one would thus have a corresponding increase of $\mathrm{Al}$ concentration and a decrease of $\mathrm{Mn}$ concentration. Although these variations cannot be detected by EDX and ICP-OES, this argument is qualitatively confirmed from the observation that annealing at the relatively low temperature of $500-600^{\circ} \mathrm{C}$ in several cases resulted in Mn-rich precipitates in the upper part of the crystals but not in their lower parts. ${ }^{11}$

In the present investigation the samples were studied in the as-grown state. EDX measurements in SEM on a polished surface did not show any compositional variation. Annealing is not expected to change any compositional gradient on the scale of the sample size.

The cylinder formed icosahedral crystal of about $10 \mathrm{~mm}$ in diameter and $10 \mathrm{~mm}$ in length was cut as illustrated in Fig. 1. The drawing is oriented in the growth direction with the top of the drawing corresponding to the part of the crystal which was first solidified. The growth direction was along a twofold symmetry axis, and samples cut in this direction are denoted by roman figures II and III, preceeded by an arabic figure for the position of the sample in the cut. Samples cut perpendicularly to the growth direction along a fivefold axis were also investigated. They are denoted A and B, and similarly with arabic figures for the position in the slice. The size of each sample was about $1 \times 1 \times 10 \mathrm{~mm}^{3}$.

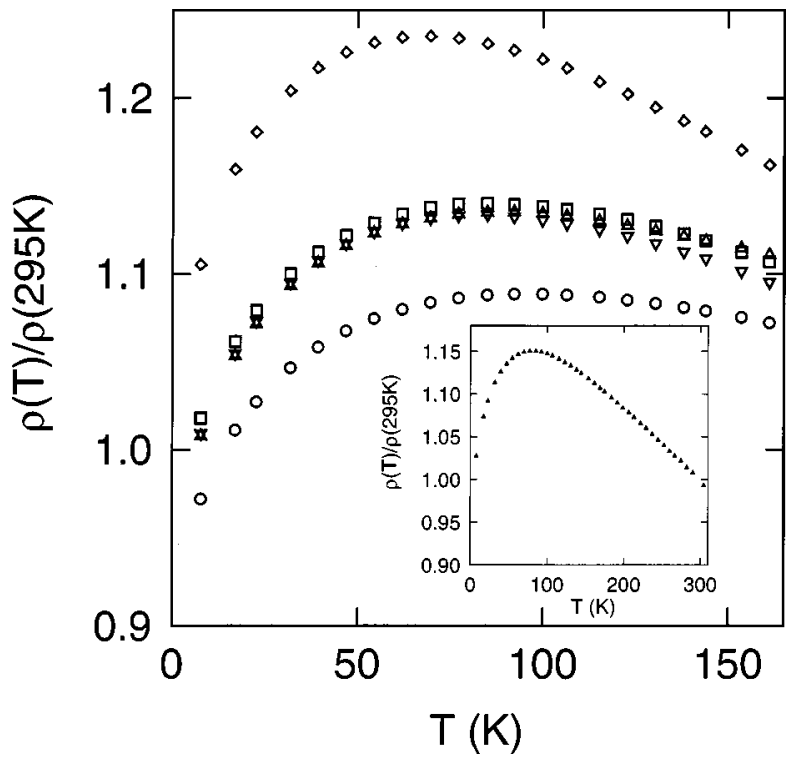

FIG. 2. The resistivity, $\rho(T) / \rho(295 \mathrm{~K})$, along the twofold axis in the 3III sample at different positions along the sample with the following symbols, position $1 \diamond, 2 \nabla, 3 \triangle, 4 \square, 5 \bigcirc$, where 1 is close to the bottom and 5 is close to the top. Note the shift in the maximum along the sample. Inset: The resistivity in the temperature range $1.5-300 \mathrm{~K}$ measured along the entire sample.

The resistance was measured by an ordinary four-probe technique. The room-temperature resistivity was about 2.4 $\mathrm{m} \Omega \mathrm{cm}$, with no systematic differences between different samples within the measurement error of $\pm 10 \%$. Two current and six voltage probes of silver paint were placed along the sample, making it possible to measure the resistance at five positions over the sample length. In this way it was possible both to measure the variation of resistance along the twofold and fivefold axes, and to correlate this result to the original position in the crystal.

The magnetoresistance was measured in a ${ }^{4} \mathrm{He}$ cryostat equipped with a 12-T superconducting solenoid. These measurements were limited to 4.2 and $1.5 \mathrm{~K}$, the pumping limit of the cryostat, and the temperatures were stable during measurements.

\section{RESULTS}

The temperature dependence of the electrical resistivity was measured along the twofold axis in the sample 3III. The results are displayed in Fig. 2 in the form of $\rho(T) / \rho(300 \mathrm{~K})$. The overall behavior with a comparatively flat maximum at intermediate temperatures of order $50-100 \mathrm{~K}$ is in agreement with previous reports on single- as well as polygrained $i$-Al-Pd-Mn. ${ }^{2-6,8}$ With some scatter for the samples at intermediate positions, it can be seen from Fig. 2 that there is a clear trend for a stronger temperature dependence and an increasing maximum in $\rho(T) / \rho(300 \mathrm{~K})$ when moving in the growth direction from top to bottom of the sample, together with a weak tendency for a decreasing temperature of the maximum resistivity in the same sequence. The temperature dependence measured along the whole sample is shown in the inset. It is close to the average of the other measurements as expected.

The average temperature dependence of $\rho(T)$ as mea- 


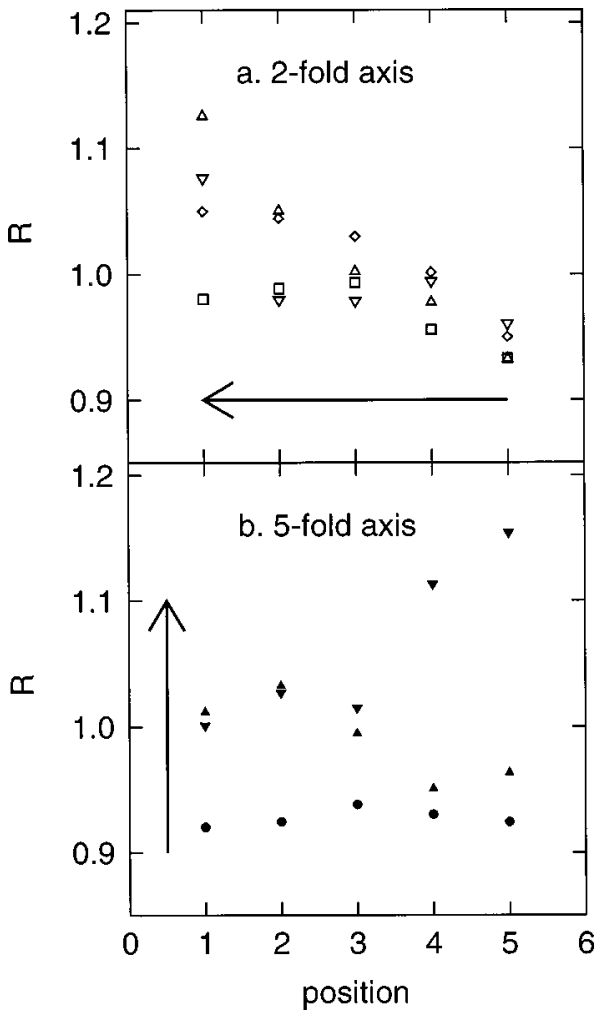

FIG. 3. The resistance ratio $R=\rho(4 \mathrm{~K}) / \rho(295 \mathrm{~K})$ vs the position for the twofold samples (a) and the fivefold samples (b). The numbers are the positions along the sample. In the twofold samples position 1 is closest to the bottom and 5 is closest to the top. In the fivefold samples the high and low position numbers do not reflect a particular side, i.e., position 5 in one sample may correspond to position 1 in another sample. The samples have the following symbols; twofold axis: $\nabla$ 3III, $\triangle$ 2III, $\diamond$ 3II, and $\square$ 2II, and fivefold axis: $5 \mathrm{~A} \boldsymbol{\nabla}, 3 \mathrm{~A} \boldsymbol{\Delta}$, and $1 \mathrm{~A} \boldsymbol{\bullet}$. In panel (a) the growth direction along the bar of the samples is indicated by the arrow and in panel (b) the ordering of the samples is along the growth direction indicated by the arrow.

sured by the resistance ratio $R=\rho(4 \mathrm{~K}) / \rho(295 \mathrm{~K})$ was determined for all samples. The results are shown in Fig. 3. For the twofold direction, position 1 is the closest to the bottom of the sample. It can be seen that $R$ increases in the direction from top to bottom of the ingot. There is approximately an $11 \pm 6 \%$ difference in $R$ between top and bottom, when averaged over all samples in the twofold direction. For the fivefold axis the $R$ value does not differ systematically along the length of the samples, i.e., in the transversal direction of the ingot. However, when moving in the sequence $1 \mathrm{~A} \rightarrow 3 \mathrm{~A} \rightarrow 5 \mathrm{~A}$, that is from a fivefold sample close to the top to a fivefold sample close to the bottom of the ingot, there is a systematic increase in $R$ in agreement with the results for the twofold direction. Consistent with the results for the twofold direction, the average $R$ value of samples $5 \mathrm{~A}$ and $1 \mathrm{~A}$ showed a difference of $14 \pm 6 \%$. No anisotropy in the temperature dependence could thus be detected. However a sizeable variation in $\rho(T) / \rho(300 \mathrm{~K})$ along the growth direction has been verified.

The magnetoresistance was measured in all samples. In Fig. $4, \Delta \rho(B, 1.5 \mathrm{~K}) / \rho(0,1.5 \mathrm{~K})$ is shown for a few samples. The magnetoresistance is positive for all fields and temperatures measured and of varying magnitude, consistent with the

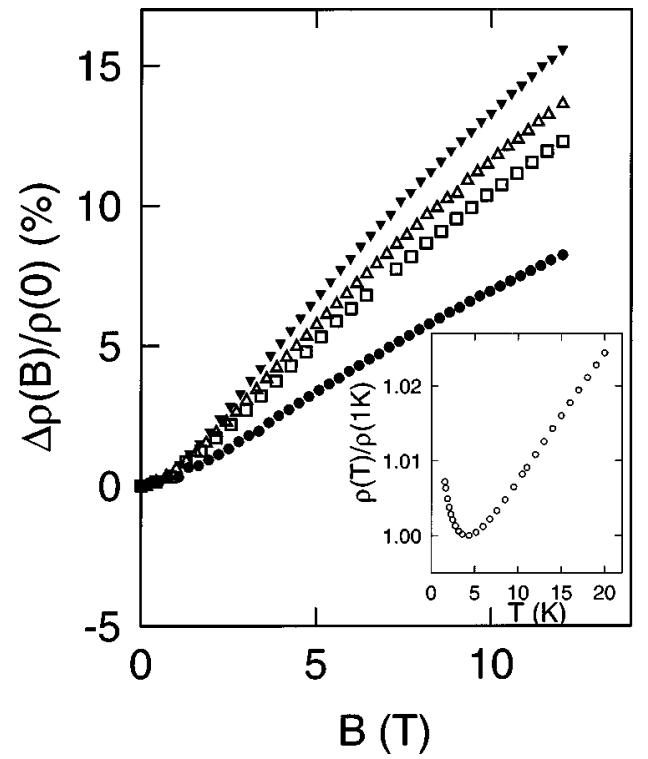

FIG. 4. Main panel: The magnetoresistance $\triangle \rho(B) / \rho(0)$ at 1.5 $\mathrm{K}$ vs magnetic field measured along the entire samples, which have the following symbols: fivefold axis: $5 \mathrm{~A} \boldsymbol{\nabla}$ and $1 \mathrm{~A} \boldsymbol{\bullet}$, twofold axis: $\triangle$ 2III and $\square$ 2II. Inset: The temperature dependence of the resistivity $\rho(T) / \rho(4 K)$ for an $\mathrm{Al}_{70.9} \mathrm{Pd}_{20.7} \mathrm{Mn}_{8.4}$ sample from the same batch as in Ref. 6.

range of results previously reported. ${ }^{3-6}$ It can be seen from Fig. 4 that there is a large difference in the MR for the samples along the fivefold axis, taken from the extreme axial positions of the studied material, while the results for the two samples along the twofold axis, taken from the transverse extremes of the crystal, are less scattered, and are encompassed by the results for the other samples.

The magnetoresistance at $12 \mathrm{~T}$ and $4 \mathrm{~K}, \Delta \rho(12 \mathrm{~T}, 4 \mathrm{~K}) /$ $\rho(0,4 \mathrm{~K})$, is shown in Fig. 5 for the same samples as in Fig. 3 as a function of position along the samples. Similar to the results for $\rho(T)$, there is a decrease in the magnitude of the MR when moving in the direction from the bottom to the top of the ingot and $\Delta \rho(12 \mathrm{~T}, 4 \mathrm{~K}) / \rho(0,4 \mathrm{~K})$ is approximately $28 \pm 6 \%$ smaller in the first solidified part of the crystal, than at the bottom. Perpendicular to the growth direction, along the fivefold samples, there is again no systematic variation of the MR. In this case however, the sample at the top, $1 \mathrm{~A}$, has a value of $\Delta \rho(12 \mathrm{~T}, 4 \mathrm{~K}) / \rho(0,4 \mathrm{~K})$ which is roughly 40 $\pm 4 \%$ smaller than for sample $5 \mathrm{~A}$ at the bottom.

\section{DISCUSSION}

There is no indication of anisotropy from the measurements of the resistance or the magnetoresistance. As for the resistance, this result is in agreement with the expectations for the resistivity tensor under the icosahedral point-group symmetry, while this argument does not exclude anisotropy in the magnetoresistance. ${ }^{12}$ The differences in $\rho(T)$ and the MR are in themselves interesting, particularly in view of the high structural quality of the samples studied. It is probable from the conditions of the growth of the crystal as described above, that these differences are due to a gradient in the $\mathrm{Mn}$ concentration. We discuss the results within this hypothesis. 


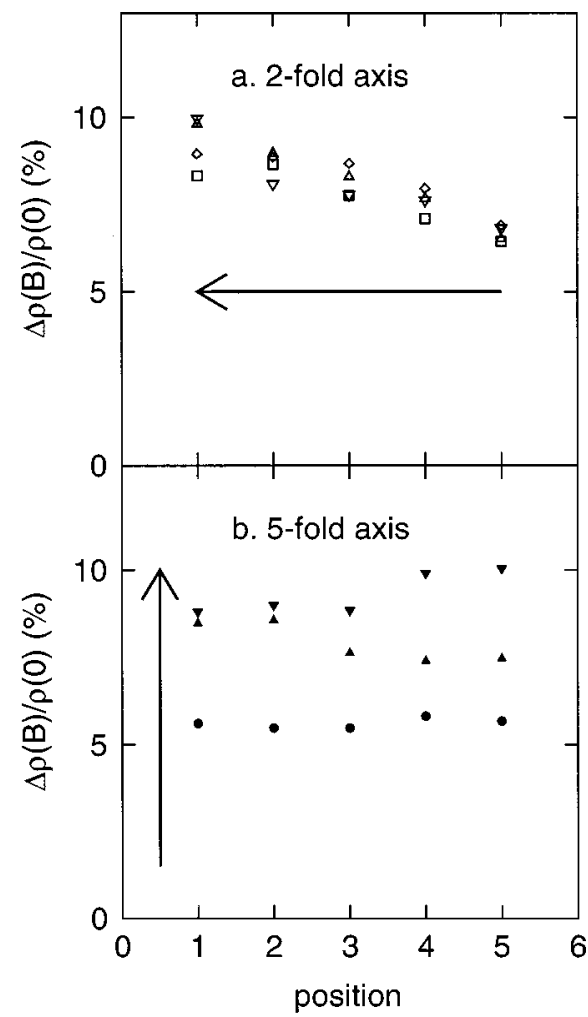

FIG. 5. The magnetoresistance at $12 \mathrm{~T}$ and $4 \mathrm{~K}$, $\triangle \rho(B=12 \mathrm{~T}, 4 \mathrm{~K}) / \rho(0,4 \mathrm{~K})$, vs the positions in the samples along the along twofold axis (a) and along the fivefold axis (b). Position numbering according to figure legend in Fig. 3, with position 1 close to the bottom and position 5 close to the top for the twofold samples, and filled circles close to the top and filled down triangles close to the bottom for the fivefold samples. In panel (a) the growth direction along the bar of the samples is indicated by the arrow and in panel (b) the ordering of the samples is along the growth direction indicated by the arrow.

\section{A. Resistivity}

The decrease of $\rho(T)$ below about $50-100 \mathrm{~K}$ is not well understood. Although work on single grained $i$-Al-Pd-Mn show similar maxima, ${ }^{2-6}$ results for polygrained samples indicate that such a maximum may depend on annealing conditions and on Mn concentration, ${ }^{3,8}$ with a tendency for this maximum to develop with decreasing Mn concentration below about 10 at. $\%$ and to be suppressed again below about 8 at. $\%$. Conduction mechanisms in this region of resistivities likely include quantum interference effects (QIE) and magnetic scattering both in the form a dephasing contribution in QIE and direct spin scattering. QIE have successfully described transport properties in icosahedral quasicrystals, e.g., polygrained $\mathrm{Al}-\mathrm{Pd}-\mathrm{Mn},{ }^{13}$ and $\mathrm{Al}-\mathrm{Cu}-\mathrm{Fe},{ }^{14}$ with precision. For single grained Al-Pd-Mn it was reported in Ref. 6 that QIE with a very strong spin-orbit scattering ( $\tau_{\text {so }} \approx 4 \mathrm{fs}$ ) could describe the magnetoresistance. A small $\tau_{\text {so }}$ is qualitatively consistent with a maximum in $\rho(T)$ in the weak localization contribution at a high temperature. The trend in Fig. 3 that the temperature at the maximum $T_{\max }$ decreases when moving from the top to the bottom of the ingot could then indicate a decreasing spin-orbit interaction strength. The spinorbit interaction increases strongly with the mass of the elements. Since the growth conditions indicate not only an increase of $\mathrm{Al}$ content, and a decrease of the $\mathrm{Mn}$ concentration along the growth direction, but also a small decrease of $\mathrm{Pd}$, it is difficult to uniquely correlate the change of the spinorbit interaction strength with the mass change. If the change of $\mathrm{Pd}$ concentration is negligible, the trend observed in $T_{\max }$ is qualitatively consistent with weak localization and a decreasing spin-orbit interaction.

A dephasing contribution from magnetic scattering would also influence the resistivity. From a calculation by Amaral ${ }^{15}$ of the corresponding contribution in weak localization, the maximum in $\rho(T)$ at $T_{\text {max }}$ is displaced towards lower temperatures for an increasing spin-scattering rate, while the observations in Fig. 2 instead show that $T_{\text {max }}$ decreases towards the bottom of the crystal, where the Mn concentration is the smallest. Since the weak localization contribution is sensitive to magnetic scattering in the icosahedral phase, while from the growth conditions we can only infer a decrease in the growth direction of the total Mn concentration of the ingot, these observations may suggest, but do not prove, that changes over the sample length of magnetic dephasing scattering do not contribute significantly to $\rho(T)$.

A Kondo effect from direct spin-scattering off magnetic impurities should also be considered. In fact, such a contribution is suggested from the characteristic feature of a lowtemperature minimum in the resistivity. A low-temperature minimum in $\rho(T)$ has been observed previously in polygrained $i$-Al-Pd-Mn samples. ${ }^{3}$ The inset in Fig. 4 shows a similar observation in a single grained sample from the same batch as studied in Ref. 6. The measured resistivity may contain contribution from all the effects mentioned.

\section{B. Magnetoresistance}

A magnetoresistance of order several percent in a material with a resistivity of several $\mathrm{m} \Omega \mathrm{cm}$ can usually be well described by quantum intereference effects. In metallic systems such as Al-Pd-Mn no other mechanism than QIE is known which can account for these observations. A magnetic impurity effect, e.g., an increase of magnetic Mn would effectively destroy the phase coherence of the electron wave functions and result in a smaller (positive) magnetoresistance, in qualitative agreement with the results in Fig. 4, where $\Delta \rho(B, T) / \rho(0, T)$ for the fivefold direction is the smallest in a sample close to the top of the ingot.

One could object to this interpretation by the following argument; Within some scatter of $10 \%$, the resistivity at room temperature is the same for all samples. There is a systematically larger increase of $\rho(T)$ with decreasing temperature for samples close to the bottom of the ingot (twofold direction, Fig. 3). The measured $\Delta \rho(B, T) / \rho(0, T)$ contains this $\rho(T)$ as a factor, and it could therefore be that it is larger close to the bottom of the ingot (e.g., twofold direction Fig. 5) due to a change in $\rho(T)$ from a magnetic effect or an effect in $\tau_{\text {so }}$, and not to the intrinsic magnetoresistivity. However, even if the entire change in $\rho(T)$ between room temperature and $4 \mathrm{~K}$, i.e., $R$ of about $11 \pm 6 \%$, were considered in this estimate, it is still only a fraction of the change of

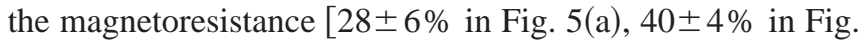
$5(\mathrm{~b})]$. Therefore the magnetoresistance is likely also affected by spin scattering.

We have tried to circumvent the problem with varying Mn concentration by measuring the magnetoresistance along 
both the fivefold and twofold axes on the same sample piece. A sample from the same batch as in Ref. 6 was reduced to a slice of $1 \times 1 \times 0.2 \mathrm{~mm}^{3}$ with the twofold and fivefold symmetry axes along the two longer sides. No anisotropy was observed in these measurements. However, one must caution that in such a small sample, current may be distributed not only along the considered symmetry direction, influencing the interpretation of this result.

Thus no anisotropy of the magnetoresistance has been detected. The large differences in MR observed between samples from different parts of the crystal are presumably due to a combined effect of resistivity changes due to magnetic impurities and an intrinsic magnetoresistance effect associated with dephasing of the weak localization contribution. The resistivity as well as the magnetoresistivity of $i$-Al-Pd-Mn are thus likely composed of a number of different contributions, and detailed analyses seem intractable. Therefore we cannot determine characteristic parameters for the Mn scattering.

It was possible to describe the magnetoresistance of similar samples with negligible spin-scattering. ${ }^{6}$ It may be that inclusion of the parameters for spin scattering renders the formalism of quantum corrections overflexible. Our present results suggest a somewhat surprising sensitivity of the magnetoresistance to minute quantities of magnetic Mn. An estimate of the content can be obtained from magnetic susceptibility measurements. Assuming an impurity magnetic moment for Mn of $4-5 \mu_{B}$, the weight fraction of magnetic Mn was estimated to be $0.1-0.2$ wt. $\%,{ }^{6}$ i.e., similar to the upper limit of manganese concentration variations in the samples. With the sample length of $1 \mathrm{~cm}$, the present measurements thus display sensitivity to concentration gradients of below 0.2 wt. $\% / \mathrm{cm}$.

\section{CONCLUDING REMARKS}

By proper selection of samples from a single grain ingot of $i$-Al-Pd-Mn, it has been possible to separate the effects of anisotropy and impurities. We can conclude first that we have found no evidence for anisotropy, neither in the temperature dependence of the resistivity nor in the magnetoresistance. The different temperature dependences of $\rho(T)$ in different samples appear to be qualitatively explained by a different $\mathrm{Mn}$ concentration in the direction of the crystal growth. It appears likely that contributions to $\rho(T)$ should be considered both from a Kondo effect and from varying spinorbit scattering and possibly also from spin scattering within weak localization. Similarly, evidence has been found that the effect observed in the magnetoresistance cannot likely be explained by resistivity changes alone, but is an intrinsic magnetoresistance effect, with contributions both from spin dephasing and direct spin scattering. The number of parameters required appear to make detailed analyses of $\rho(T)$ and $\Delta \rho(T) / \rho(T)$ unfeasible. However, clear indications have been found that $\mathrm{Mn}$ concentration gradients influence the result at a level which is below the detection limit in usual chemical analyses methods. Our results thus emphasize that the magnetoresistance is a quite sensitive probe for sample control.

\section{ACKNOWLEDGMENTS}

Stimulating discussions with C. Berger have been very fruitful. The authors thank $\mathrm{C}$. Thomas for technical contributions. This work has been supported by the Swedish Natural Science Research Council and by EC Contract No. ERBCHRXCT940528.
*Present address: Solid State Division, Bldg. 3025 ORNL, PO Box 2008, Oak Ridge, TN 37831-6030.

${ }^{1}$ D. Shechtman, I. Blech, D. Gratias, and J. W. Cahn, Phys. Rev. Lett. 53, 1951 (1984).

${ }^{2}$ Y. Yokoyama, T. Miura, A.-P. Tsai, A. Inoue, and T. Masumoto, Mater. Trans., JIM 33, 97 (1992).

${ }^{3}$ H. Akiyama, T. Hashimoto, T. Shibuya, K. Edagawa, and S. Takeuchi, J. Phys. Soc. Jpn. 62, 939 (1993).

${ }^{4}$ K. Saito, S. Matsuo, H. Nakano, T. Ishimasa, and M. Mori, J. Phys. Soc. Jpn. 63, 1940 (1994)

${ }^{5}$ M. Rodmar, M. Ahlgren, C. Berger, A. Sulpice, M. Beyss, N. Tamura, K. Urban, and Ö. Rapp, Czech. J. Phys. 46, 2703 (1996).

${ }^{6}$ M. Rodmar, M. Ahlgren, C. Berger, A. Sulpice, M. Beyss, N. Tamura, K. Urban, and Ö. Rapp, in Proceedings of the 6th International Conference on Quasicrystals, edited by S. Takeuchi and T. Fujiwara (World Scientific, Singapore), p. 692.

${ }^{7}$ K. Fukamichi, in Physical Properties of Quasicrystals, edited by Z. Stadnik Springer Series in Solid State Physics Vol. 126, (Springer, Berlin, 1999), p. 295.

${ }^{8}$ P. Lanco, T. Klein, C. Berger, F. Cyrot-Lackmann, G. Fourcaudot, and A. Sulpice, Europhys. Lett. 18, 227 (1992).

${ }^{9}$ E.g., D. Hurle, Crystal Pulling fom the Melt (Springer, Berlin, 1993).

${ }^{10}$ T. Gödecke and R. Lück, Z. Metallkd. 86, 2 (1995).

${ }^{11}$ B. Grushko, N. Tamura, and K. Urban (unpublished).

${ }^{12}$ C. Berger and P. Kalugin (private communication).

${ }^{13}$ P. Lindqvist, P. Lanco, C. Berger, A. G. M. Jansen, and F. CyrotLackmann, Phys. Rev. B 51, 4796 (1995).

${ }^{14}$ M. Ahlgren, P. Lindqvist, M. Rodmar, and Ö. Rapp, Phys. Rev. B 55, 14847 (1997).

${ }^{15}$ V. S. Amaral, J. Phys.: Condens. Matter 2, 8201 (1990). 